\title{
CrystEngComm
}

Check for updates

Cite this: CrystEngComm, 2021, 23, 252

Received 27th August 2020

Accepted 23rd September 2020

DOI: $10.1039 / \mathrm{d} 0 \mathrm{ce} 01260 \mathrm{~b}$

rsc.li/crystengcomm

\section{Identification of synthesisable crystalline phases of water - a prototype for the challenges of computational materials design}

\author{
Edgar A. Engel (1D
}

\begin{abstract}
We discuss the identification of experimentally realisable crystalline phases of water to outline and contextualise some of the diverse building blocks of a computational materials design process. The example of water ice allows us to highlight important challenges and to discuss recent steps towards their resolution. Starting with an extensive database-driven computational search for (meta-)stable crystalline phases, we use dimensionalityreduction techniques to visualise and rationalise the configuration space of ice, screen for promising candidates for thermodynamic stability, and, finally, touch upon accurate, predictive determination of relative stabilities. We conclude by highlighting some of the open problems in practical computational materials design.
\end{abstract}

\section{Introduction}

The following discusses and contextualises work published over the recent years. In particular, it highlights and synthesises the work published in refs. 55, 87, 115 and 137. It does not contain any novel work. Since the first calculations in statistical physics, ${ }^{1}$ computer simulations have cemented themselves as an integral part of the physical sciences. In materials science the field of computational materials design (CMD) has profited particularly from Moore's law and computing architectures tailored towards big data applications. CMD promises to accelerate the discovery and design of novel, technologically interesting materials. With materials as the catalyst for incisive technological (and societal) developments, CMD promises to actively change the world we live in.

In the following, we set aside computationally aided but experimentally driven materials discovery despite its unquestionable value: whether it is the computational identification of the atomic structure of an experimentally discovered phase, or materials design by means of tweaking an established class of structure in terms of dopants/ composition/stress/etc.

With this caveat the potential of CMD has arguably not been realised yet. Its greatest value - the ability to characterise structures and materials at a rate that exceeds that of experiment by orders of magnitude - is also its greatest weakness, since CMD easily overwhelms experimental capacities for syntheses and validating predictions. The variable predictive power of CMD studies and a preference for comparatively simple and/or well

TCM Group, Cavendish Laboratory, University of Cambridge, J. J. Thomson Avenue, Cambridge CB3 OHE, UK. E-mail: eae32@cam.ac.uk established materials compound this issue. The remainder of this highlight article will be a prime example.

Numerous studies such as ref. 2-8 demonstrate the efficient computational generation of novel structures by combining atomistic calculations with structure searching techniques, ${ }^{9-15}$ but the fewest result in the synthesis of a novel material. In order to understand the reasons for this inefficiency, it is worth outlining the canonical CMD workflow. CMD starts with a search of the space of possible (meta-)stable structures. Their number inevitably requires distilling promising candidates, before predictive assessments of thermodynamic stability and properties can identify structures, for which is worthwhile to establish possible synthesis pathways. Real CMD workflows are substantially less streamlined and may be constructed from a variety of building blocks, as schematically illustrated in Fig. 1. This renders CMD complex, material-specific, and labour- and expertise-intensive, making the integration of the above building blocks into a unified, accessible framework the key to computationally driven discovery of technologically relevant materials.

In view of this complexity, it is worthwhile juxtaposing an outline of the "canonical" workflow with that described in the following. The arguably most widespread workflow involves generating atomic or molecular configurations from scratch (or by chemical substitution), and subsequently determining their configurational energies using molecular force fields or density functional theory (DFT). Structures are then screened according to their properties and/or configurational energies. In contrast, in the following we describe a database-driven approach to generating ice structures, which are then geometry optimised using DFT and globally screened for thermodynamic stability using a generalised convex hull construction. Crucially, extensive and 


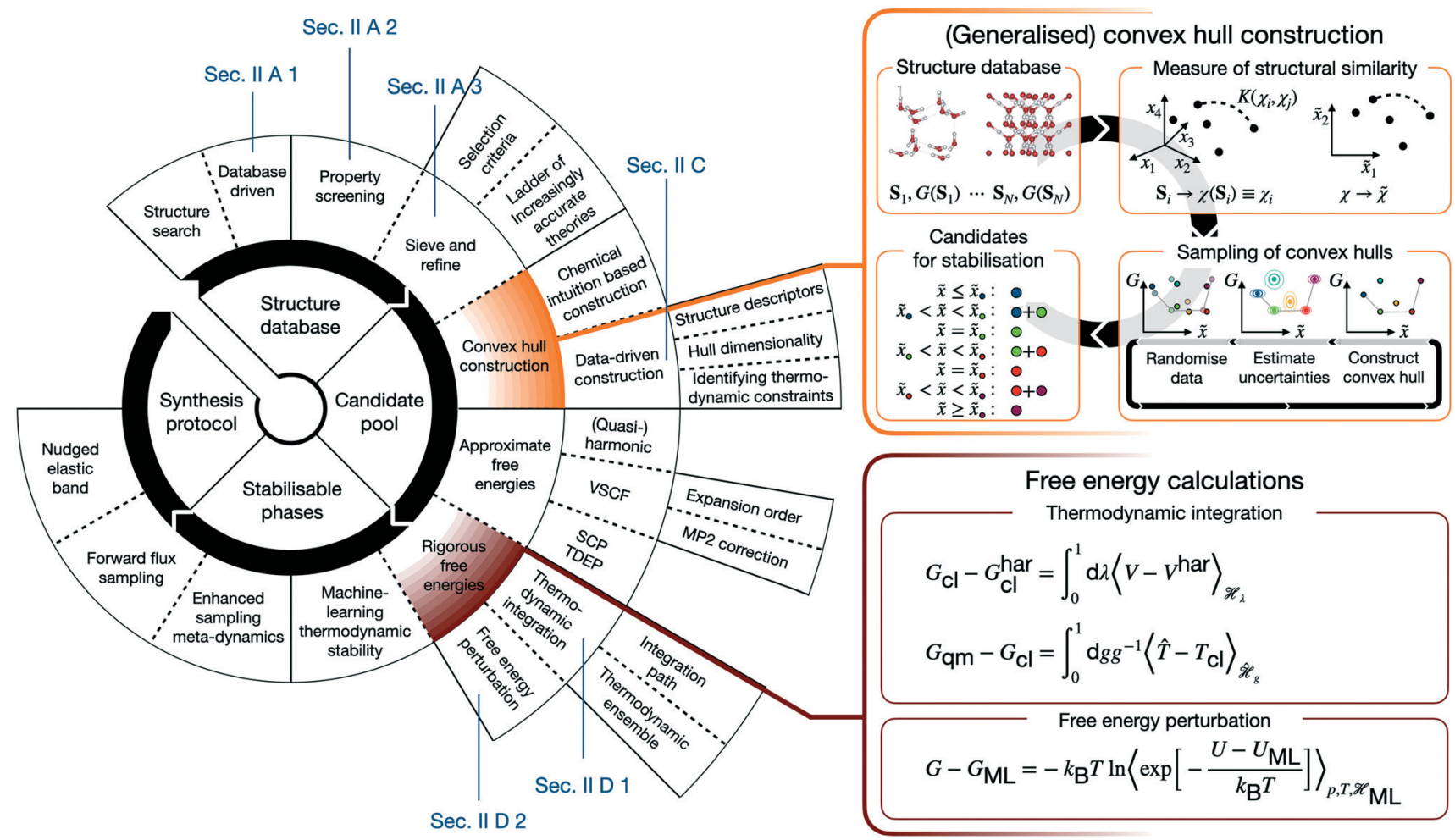

Fig. 1 Schematic overview of a CMD workflow highlighting the variety of approaches (left panel), and more detailed views of the constituent steps/equations of the GCH construction for screening structure data (top right panel, section II C) and rigorous free energy methods for assessing thermodynamic stability (bottom right panel, section II D).

rigorous free energy calculations put our understanding of thermodynamic stability on a firm footing. Both canonical approaches and the subsequently described one are usually followed by further characterisation of the most promising candidates.

Using the example of identifying crystalline phases of water with the thus outlined workflow, we illustrate some of the more recent developments in (i) screening of structure data and (ii) free energy methods for high-throughput applications, we review the process of identifying crystalline phases of water. Much of the computational detail is brushed under the carpet in order to leave a clearer view of how methodological developments may be married together in a CMD workflow.

\section{Water ice as a case study}

Despite its apparent simplicity water exhibits phenomena, which are characteristic of multiple classes of materials. Proton (dis-)order is an instance of configurational disorder, otherwise observed in materials such as doped semiconductors $^{16-18}$ and high-entropy alloys, ${ }^{19}$ while the molecular nature of ice leads to features that are characteristic of molecular systems in general: anomalous behaviour relating to thermal expansion ${ }^{20}$ and isotopic substitution $^{21,22}$ reveals the importance of nuclear quantum effects (NQE). Polymorphism and hydrogen bonding and dispersion interactions play important roles, just like in pharmaceuticals ${ }^{23-26}$ and layered materials, such as graphene-hBN superlattices. ${ }^{27}$ Properties and thermodynamic stability can be tuned by isotopic substitution, ${ }^{28-30}$ reminiscent of e.g. other molecular crystals. ${ }^{31-33}$ Last but not least, the phase-diagram of water prominently highlights the importance of meta-stability: seven of 18 experimentally characterised crystalline phases ${ }^{34}$ are metastable. $^{35}$

Water and ice are some of the most extensively studied systems in the physical sciences, and have been investigated across a wide range of temperatures and pressures. For the purpose of this exercise, we will assume our knowledge to be limited to (i) the liquid form and (ii) its propensity for forming four-connected, tetrahedral networks following the "Bernal-Fowler ice rules". ${ }^{36}$ We set aside all further understanding of the phase-diagram to highlight to which extend decades of experimental and theoretical work could be reproduced in a single, state-of-the-art, computational exploration of the phase-space of water.

\section{A. Survey of locally-stable crystal structures}

Searching for (meta-)stable phases implies exploring phasespace, for instance using molecular dynamics (MD), nested sampling, ${ }^{37}$ or other algorithms. MD approaches range from plain (path-integral) MD to minimum hopping, ${ }^{11}$ (temperature) replica-exchange $\mathrm{MD}$, multithermal-multibaric ensembles, $^{38}$ and enhanced sampling meta-dynamics. ${ }^{39,40}$ 
While such approaches can directly provide thermodynamic stabilities, they do not lend themselves to high-throughput applications. Consequently, extensive structure searches typically explore configuration-space and only assess local stability in terms of their potential energy. A history of crystal structure predictions highlights the potential of diverse

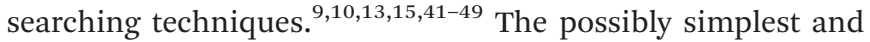
most elegant approach is to generate many different random arrangements of atoms and performing a local potential energy minimisation with respect to the atomic positions (and lattice parameters for periodic configurations) using a first-principles electronic-structure method such as DFT. ${ }^{9}$

1. Database-driven structure generation. However, for ice one may instead take inspiration from searches for $\mathrm{sp}^{3}$ allotropes of carbon ${ }^{50}$ and ultralow-density ices, ${ }^{49}$ and forego explicit structure prediction in favour of exploiting the isomorphism between ice and silica networks. ${ }^{51-55}$ There is a vast literature on aluminosilicates, including a database of experimentally confirmed structures ${ }^{56}$ and different databases of theoretically-enumerated networks, such as those of Treacy $^{57}$ and Deem. ${ }^{58}$ These constitute a comprehensive source of more than five million fourconnected networks from which one can generate topologically-distinct polymorphs of ice by placing oxygen atoms on the vertices and hydrogen atoms on the midpoints between neighbouring vertices. Their size necessitates some preselection of structures.

2. Property screening. A pragmatic, systematically improvable preselection strategy is to limit the unit cell size. In ref. 5574731 structures $\dagger$ with unit cell volumes up to 800 $\AA^{3} \ddagger$ are supplemented with the energetically favourable, low density structures from the IZA database ${ }^{59}$ of experimentally synthesised aluminosilicates, for which energies and densities correlate with their ice counterparts. ${ }^{51}$

3. Sieve and refine. After an initial geometry optimisation of the resulting 74963 structures using the ReaxFF force field, ${ }^{60}$ removal of duplicates and high-energy configurations, $\S$ the remaining 15882 distinct structures are then refined using DFT variable-cell geometry optimisations with the $\mathrm{PBE}^{62}$ functional.

The size of this dataset reflects a key challenge of CMD: the number of locally-stable structures inevitably renders the identification of distinct, synthesisable structures a needlein-a-haystack problem. Setting aside kinetic effects, a rigorous analysis of experimental relevance requires exploring the phase-diagram not just as a function of temperature and pressure, but also electric fields, ${ }^{63}$ doping, ${ }^{64}$ isotopic substitution, ${ }^{28-30}$ presence of guest molecules, ${ }^{65-68}$ and other

\footnotetext{
$\dagger$ This selection contains duplicates since the databases are not mutually exclusive.

\$ And without 3-rings, which would normally induce excessive strain in an ice structure.

$\S$ In practice, structures with configurational energy exceeding that of an energyvolume convex hull by a multiple of the free energy differences arising from different proton-ordering and $\mathrm{NQE}$ of around $10 \mathrm{meV}$ per $\mathrm{H}_{2} \mathrm{O}$ (ref. 61) were eliminated.
}

thermodynamic constraints. Since this is not computationally viable for large numbers of structures, it becomes crucial to (i) rationalise the space of locally-stable structures, and (ii) distill structures whose favorable energetics and/or particular structural features provide leverage for stabilisation at conditions different from those of the search.

\section{B. Rationalising configuration space}

A two-dimensional representation of the similarity of the structures (see Fig. 2) provides a human-readable visualisation of structure space and an aid in developing an intuitive understanding of structural relationships, such as proton-(dis-)order, stacking faults, and two- and threedimensional periodicity.

Any such representation depends on the underlying measure of structural similarity. The field of properties regression for atomistic systems has gifted us with a variety of ways of encoding atomic positions (and unit cells) $\mathbf{S}_{\mathrm{i}}$ of structure $i$ in feature vectors/descriptors $\mathbf{X}\left(\mathbf{S}_{\mathrm{i}}\right)$, whose components are individual features $\chi$. Descriptors that remain invariant under changes of representation of the same periodic structure (e.g. due to changes in particle labelling or a different choice of unit cell) and under translations and rigid rotations of the structure are particularly suited to structure comparisons in terms of a kernel measure of similarity, || such as:

$$
K\left(\mathbf{S}_{\mathrm{i}}, \mathbf{S}_{\mathrm{j}}\right)=\left(\mathbf{X}\left(\mathbf{S}_{\mathrm{i}}\right) \cdot \mathbf{X}\left(\mathbf{S}_{\mathrm{j}}\right)\right)^{\xi}
$$

Prominent examples of approaches for translating $\mathbf{S}_{\mathrm{i}}$ into $\mathbf{X}\left(\mathbf{S}_{\mathrm{i}}\right)$ are the Coulomb matrix,$^{70}$ bag-of-bonds, ${ }^{71}$ aSLATM, ${ }^{72}$ atom-centered symmetry functions $(\mathrm{SF}),{ }^{73}$ and the smooth overlap of atomic positions (SOAP). ${ }^{74}$ The similarity map in the lefthand panel of Fig. 2 is based on an entropyregularized matching (REMatch) kernel $^{75}$ combined with a SOAP description, whose construction and parameters were designed to be insensitive to proton disorder and hydrogenbonding defects. SOAP belongs to the variety of atom-density based descriptions. ${ }^{76}$ It encodes two- and three-body correlations between atomic positions, which do not generally suffice for a unique map between structure and descriptor space. Consequently, kernel plays an important role in enhancing the effective body-order of the similarity measure and its ability to resolve structures. ${ }^{77}$

Given a similarity measure, a variety of dimensionality reduction algorithms can be employed to extract a twodimensional representation aimed at optimally reproducing the distances between pairs of structures. Both linear projections, such as principal components analysis (PCA), ${ }^{78}$ and non-linear embeddings, such as kernel PCA, ${ }^{79} \mathrm{UMAP}^{80} \mathrm{t}$ $\mathrm{SNE},{ }^{81}$ and sketch-map ${ }^{82}$ have their merits.

I Recent covariantly transforming variants permit predictions of tensorial properties. $^{69}$

\| Which is at this point defined in the high-dimensional space spanned by the components of the feature vector. 

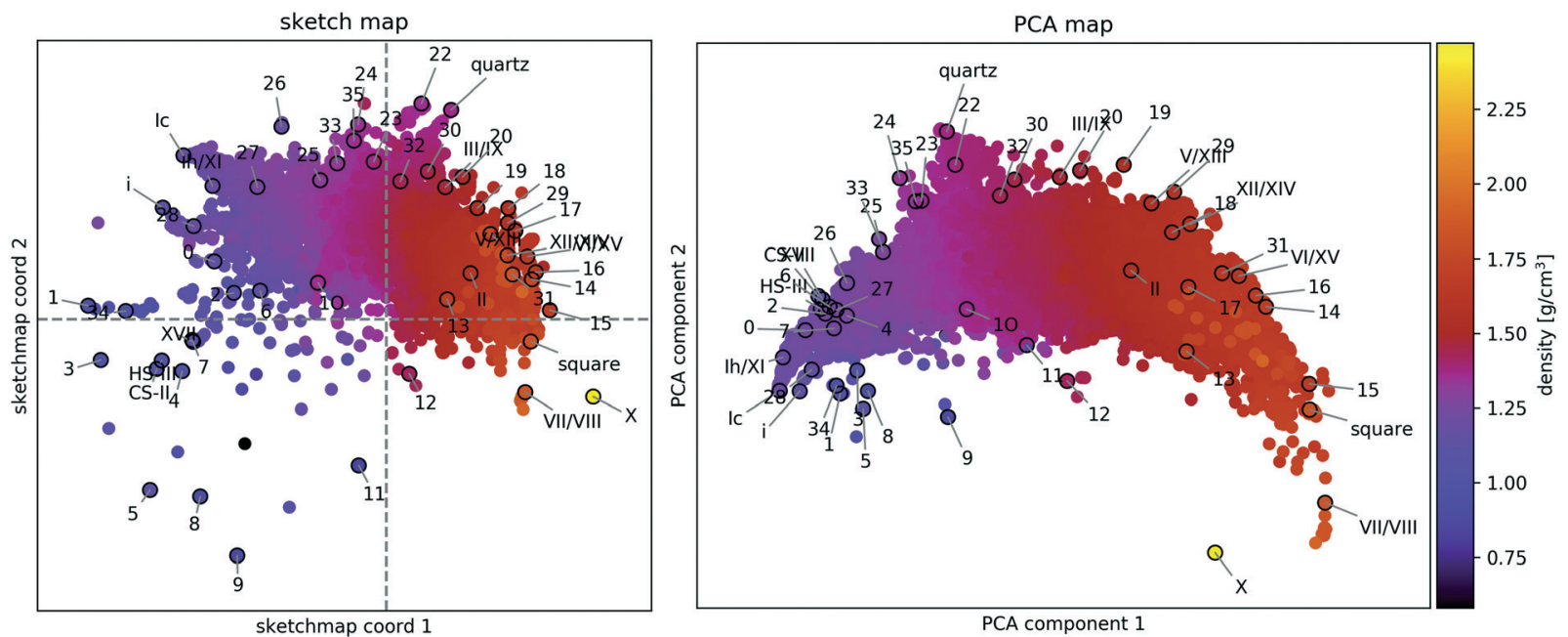

Fig. 2 Structural similarity of 15882 distinct PBE-DFT geometry-optimised ice structures. The sketch-map coordinates (left panel) and PCA (right panel) principal components (PC) are abstract measures of structural features. Hence their numerical value is not indicated. The mass density of each structure is encoded by the colour of the respective point and the known phases of ice and the 34 candidates from ref. 55 are labelled according to the original scheme.

A sketch-map of the 15882 ice structures is shown in the lefthand panel of Fig. 2, while the righthand panel shows a PCA projection of the same data. Reassuringly, the overall distribution of structures is consistent with the search strategy. The upper sector, corresponding to tetrahedral ices and silica-like networks is densely sampled, while the lower sector, corresponding to very dense (right) and very open structures (left), is sparse. At high density, this sparsity is due to geometric constraints limiting the number of pure phases and the absence of (phase-separated) mixtures, for which interfacial regions lead to reduced density. Meanwhile, at low density this sparsity arises from the preselection strategy, making the latter the biggest limitation to the comprehensiveness of the survey.

The observation that Fig. 2 is consistent with our understanding of the structure data** validates the underlying similarity measure and its use in evaluating a data-driven indicator of thermodynamic stability.

\section{Screening for stabilisable structures}

Stabilising structures, which are un-/meta-stable at the conditions of the survey, relies on exploiting structural features to manipulate the relative stability of structures. For instance, by increasing pressure one can stabilise more dense structures with respect to less dense ones, while by pumping guest molecules such as $\mathrm{H}_{2}$, methane, or carbon-dioxide into ice networks one can achieve the opposite effect.

Assuming that the Gibbs free energy $G$ depends linearly on a structural feature $\chi$ such as molar volume.

$$
G=G_{0}+\Phi \chi
$$

\footnotetext{
** Structures related by proton-disorder (which permits extracting one protonordered representative per cluster) and those related by stacking disorder are clustered together.
}

The thermodynamically stable structures at different values of a thermodynamic constraint $\Phi$ constitute the easily computed vertices of the convex hull $(\mathrm{CH})$ of $G(\chi)$, which encloses all pairs $G_{\mathrm{i}}, \chi_{\mathrm{i}}$ corresponding to the structures $\mathrm{i}$ in the dataset (see Fig. 3).

In the macroscopic limit, where interfacial energy costs become negligible, all other phases are unstable to decomposition into phase-separated mixtures of "vertex structures" at fixed $\chi$. The $\mathrm{CH}$ construction generalises to more than one feature, considering $G(\mathbf{X})$ instead of $G(\chi)$, and is routinely performed for concentrations of multiple chemical species. ${ }^{83}$ In practice kinetic effects may suppress decomposition almost indefinitely, ${ }^{84}$ but (as we will argue in section II D) thermodynamic stability is still the key indicator for synthesisability.

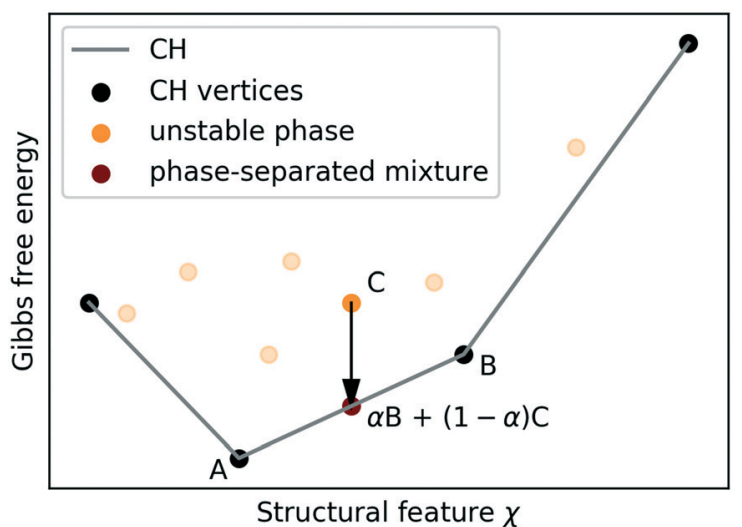

Fig. 3 Toy example illustrating the construction of a convex hull $(\mathrm{CH})$ and how structures above the $\mathrm{CH}$ (orange), such as $\mathrm{C}$ are thermodynamically unstable and will (subject to kinetic barriers) decompose into phase-separated mixtures (maroon) at fixed feature $\chi$ to lower the Gibbs free energy. 
Conventionally, $\mathrm{CH}$ are constructed almost exclusively on composition and atomic/molar volume. ${ }^{2-8}$

Choosing which feature(s) to construct a $\mathrm{CH}$ on effectively amounts to choosing a stabilisation mechanism and specifying which experimental boundary condition $\Phi$ shall be adjusted to stabilise different vertices. In general, this limits which stabilisable structures are identified. For instance, an molar-volume based $\mathrm{CH}$ identifies various pressure-stabilised ice structures (and clathrate hydrates, whose stability is subject to the presence of guest molecules ${ }^{66-68}$ ), but does not reveal phases whose synthesis requires electro-freezing, ${ }^{63}$ geometric constraints, ${ }^{85,86}$ etc.

A generalised convex hull $(\mathrm{GCH})$ construction follows a data-driven approach to feature selection. ${ }^{87}$ Here it is constructed on the same features as the map in the righthand panel of Fig. 2, where the features are linearly decorrelated using a PCA projection,

$$
\mathbf{X} \rightarrow \tilde{\mathbf{X}}=\mathbf{U X}
$$

which ensures that the features and energies of phaseseparated mixtures $A=\alpha B+(1-\alpha) C$ remain additive,

$$
\begin{aligned}
G(A) & =\alpha G(B)+(1-\alpha) G(C) \\
\tilde{\mathbf{X}}(A) & =\alpha \tilde{\mathbf{X}}(B)+(1-\alpha) \tilde{\mathbf{X}}(C),
\end{aligned}
$$

and consistent with the concept of phase decomposition. If a GCH is constructed on the fewest principal components $\tilde{\mathbf{X}}$ that retain the variance of the dataset,$\dagger \dagger$ the resultant pool of candidates still reflects the full diversity of locally-stable structures.

This inclusiveness comes at a price: the abstract nature of the principal components $\tilde{\mathbf{X}}$ requires correlating them with more intuitive properties such as density and composition to understand if/which experimentally realisable conditions may be leveraged to stabilise different vertices (or "candidates"). Fortunately, the pool of vertices is typically orders of magnitude smaller than the underlying structure database, greatly simplifying such analyses.

In principle, the $(\mathrm{G}) \mathrm{CH}$ construction assumes not only assumes eqn (2) but also the availability of exact Gibbs free energies, $G$. In practice, neither $G(\tilde{\mathbf{X}})$ nor lattice parameters and atomic positions are known exactly, rendering the $(\mathrm{G}) \mathrm{CH}$ probabilistic in nature. While this is neglected in ref. 55, there are practical benefits to a rigorous, probabilistic treatment of the $(\mathrm{G}) \mathrm{CH}$.

Since the uncertainties in lattice parameters and atomic positions propagate to the $(\mathrm{G}) \mathrm{CH}$ in a non-trivial way due to the mapping to features $\tilde{\mathbf{X}}$, it is convenient to Monte-Carlo sample $\mathrm{CHs}$ based on free energies and geometries, which are repeatedly randomised according to their respective uncertainties.

$\dagger$ The decay of the eigenvalues provides indication of the intrinsic dimensionality of the dataset. ${ }^{88}$
Importantly, very similar structures (for example owing to stacking faults or partial disorder) compete for stability and acquire small individual probabilities of constituting a vertex. This renders it possible to reduce them to a single representative structure for further analysis, by iteratively eliminating the $N$ lowest probability candidates with a cumulative probability less than one (which guarantees that no cluster is eliminated entirely in one step) from the dataset and resampling the $\mathrm{GCH}$ for the thus reduced dataset.

The probabilistic approach also significantly reduces the sensitivity to errors in input energies compared to conventional deterministic $\mathrm{CH}$ constructions. ${ }^{87}$

\section{Thermodynamic stability}

Given the evident role of kinetics in experimental syntheses, ${ }^{84}$ it is worthwhile justifying the subsequent focus on thermodynamic stability. Database analysis shows that experimentally observed metastable phases other than explosives are typically less than $200 \mathrm{meV}$ per atom away from thermodynamic stability, ${ }^{89}$ begging the question whether experimentally observed metastability is a remnant of thermodynamic stability at some other thermodynamic conditions $^{89}$ - a hypothesis which is supported by the observation that many of the meta-stable/stabilisable structures identified in section II C match the (experimentally) known ice phases and clathrate hydrates. It has further been argued that the chances of synthesising a structure increase with the associated phase-space volume. ${ }^{90}$ Free energy is thus still deemed the central indicator of synthesisability.

Within the Born-Oppenheimer (BO) approximation ${ }^{91}$ reliable, quantitative predictions of free energies require an accurate description of the electronic structure and resultant $\mathrm{BO}$ potential energy surface (PES), and the rigorous treatment of the statistical mechanics of anharmonic quantum nuclear fluctuations. While the PES can arguably be calculated routinely and accurately by first-principles methods for any conventional atomic configuration, ${ }^{92-101}$ the computational cost of extensive sampling of the nuclear degrees of freedom with first-principles methods has promoted affordable, approximate descriptions of nuclear fluctuations. Indeed, water and ice have been studied invoking a variety of approximations to both electronic structure and nuclear fluctuations, including simple electrostatic dipole models for the energetics of proton-ordering, ${ }^{102}$ force-field (PI) MD studies, ${ }^{20,103-106}$ and first-principles quasi-harmonic $(\mathrm{QHA})^{20,107}$ and vibrational self-consistent field (VSCF) ${ }^{61}$ studies. These have greatly advanced our understanding of the nature of water and ice. In particular, they have helped to (qualitatively) disentangle the roles of NQE, proton-disorder, and vibrational anharmonicity, and to understand the associated energy scales. They further show that predicting the thermodynamic stability of general ice polymorphs requires sub-meV per molecule accuracy, ${ }^{61}$ which unfortunately cannot be guaranteed with common approximate free energy methods, ${ }^{108}$ but require rigorous PI-based approaches, such as thermodynamic integration (TI). ${ }^{109-113}$ 


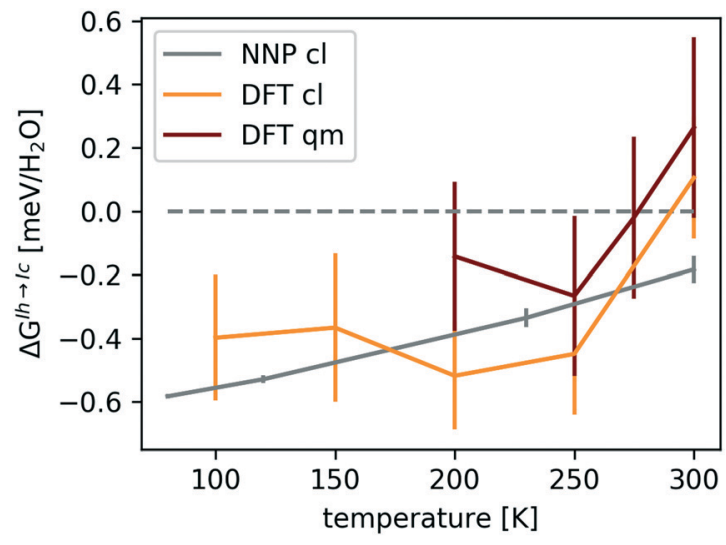

Fig. $4 \Delta G^{\mathrm{lh} \rightarrow \mathrm{Ic}}(T)$ with error bars at ambient pressure. The errors associated with the classical (cl) and quantum-mechanical (qm) revPBEO-D3 values arise predominantly from differences in $\Delta G_{N N}$ between proton-orderings. The smaller errors in $\Delta G_{\mathrm{cl}, \mathrm{NNP}}^{\mathrm{lh} \rightarrow \mathrm{Ic}}(T)$ are due to the larger simulation cell used to obtain it. The data was taken from ref. 115.

Their computational cost has previously rendered the required first-principles PI calculations impractical for any but the smallest systems, but sophisticated and affordable surrogate ML potentials have started facilitating extensive PI simulations with first-principles accuracy for more complicated systems, including water and ice. ${ }^{106,114,115}$

1. Thermodynamic integration. For instance, in ref. 115 the relative stability of the hexagonal and cubic forms of ice is determined using DFT calculations with the hybrid revPBE0 (ref. 116-118) functional and a Grimme D3 dispersion correction, ${ }^{118,119}$ capable of accurately reproducing the structure, dynamics, and spectroscopy of liquid water. ${ }^{120}+t$ The key is the use of a ML potential as a surrogate for unaffordable DFT calculations during extensive statistical sampling of nuclear fluctuations. While the limits of ML models based on fixed reference data are inevitable exceeded in configuration- and phase-space explorations targeting novel configurations and phases, this type of "sophisticated interpolation" is ideally suited to the repetitive sampling of nuclear fluctuations. $\S$

The ML potential of choice is a Behler-Parrinello type, artificial neural network potential (NNP), ${ }^{73,129,130}$ trained on reference data for 1593 diverse, 64-molecule structures of liquid water. ${ }^{115}$ It is first used to determine the classical free energy difference between ice Ih and Ic for the surrogate PES,

\$ revPBE0-D3 predicts a difference in lattice energy between the most stable proton-ordered forms of ice Ic and Ih of $U^{\mathrm{Ic}}-U^{\mathrm{Ih}}=-0.3 \mathrm{meV}$ per $\mathrm{H}_{2} \mathrm{O}, U^{\mathrm{Ic}}-U^{\mathrm{Ih}}$ $=-0.3 \mathrm{meV} / \mathrm{H}_{2} \mathrm{Oin}$ good agreement with results from diffusion Monte Carlo of $U^{\text {Ic }}-U^{\text {Ih }}=-0.4 \pm 2.9 \mathrm{meV}$ per $\mathrm{H}_{2} \mathrm{O}$ (ref. 121) and the random phase approximation of $-0.2 \mathrm{meV}$ per $\mathrm{H}_{2} \mathrm{O}$ (ref. 121) and $0.7 \mathrm{meV}$ per $\mathrm{H}_{2} \mathrm{O}^{122}$

$\S \S$ Notably, uncertainty estimation ${ }^{123}$ and active learning ${ }^{124,125}$ have paved the way for the use of ML approaches also in configuration- and phase-space exploration. Beyond regression, dimensionality-reduction techniques have proven useful in rationalising phase-spaces ${ }^{75}$ and identifying critical structural features, ${ }^{126}$ and imputation in dealing with inhomogeneous/incomplete datasets. ${ }^{127,128}$
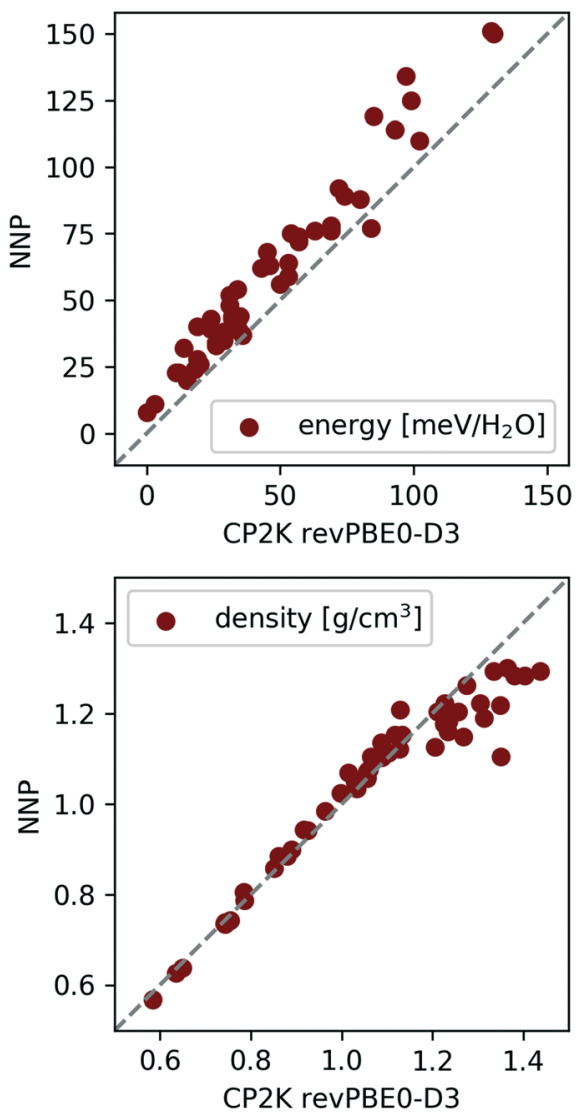

Fig. 5 Correlation between NNP and revPBEO-D3 energies (top) and densities (bottom) for the 53 ice polymorphs from section II C. The data was taken from ref. 137.

$\Delta G_{\mathrm{cl}, \mathrm{NNP}}^{\mathrm{Ih}, \mathrm{Ic}}$, by means of TI from a Debye crystal to classical ice at $25 \mathrm{~K}$ in the NVT ensemble and a transitions to the NPT ensemble to evaluate the temperature dependence of $\Delta G_{\mathrm{cl}, \mathrm{NNP}}^{\mathrm{Ih} \rightarrow \mathrm{Ic}}$ between $25 \mathrm{~K}$ and $300 \mathrm{~K} \cdot{ }^{131}$ NQEs are then taken into account by integrating the quantum centroid virial kinetic energy $\left\langle T_{\mathrm{CV}}\right\rangle$ with respect to the fictitious "atomic" mass from the classical to the quantum-mechanical limit. ${ }^{106,114,132,133}$

2. Free energy perturbation. Inevitably, the limitations of the reference data and stochastic nature of the NNP training lead to residual errors with respect to the first-principles reference. We therefore promote $\Delta G^{\mathrm{Ih} \rightarrow \mathrm{Ic}}$ to the reference level of theory by free energy perturbation (FEP), which renders $\Delta G^{\mathrm{Ih} \rightarrow \mathrm{Ic}}$ independent of the NNP. For each polymorph the Gibbs free energy at the reference level is calculated as

$$
G(p, T)=G_{\mathrm{NN}}(p, T)+k_{\mathrm{B}} T \ln \left\langle\exp \left[-\frac{U-U_{\mathrm{NNP}}}{k_{\mathrm{B}} T}\right]_{p, T, \mathcal{H}_{\mathrm{NNP}}}\right\rangle
$$

where $U$ and $U_{\text {NNP }}$ denote the reference and surrogate NNP potential energies, and $\langle\ldots\rangle_{p, T, \mathcal{H}_{\mathrm{NNP}}}$ denotes the ensemble average at temperature $T$ and pressure $p$ using the surrogate NNP Hamiltonian $\mathcal{H}_{\text {NNP }}$. During FEP $U$ is explicitly calculated using the first-principles reference method, but, with a sufficiently 

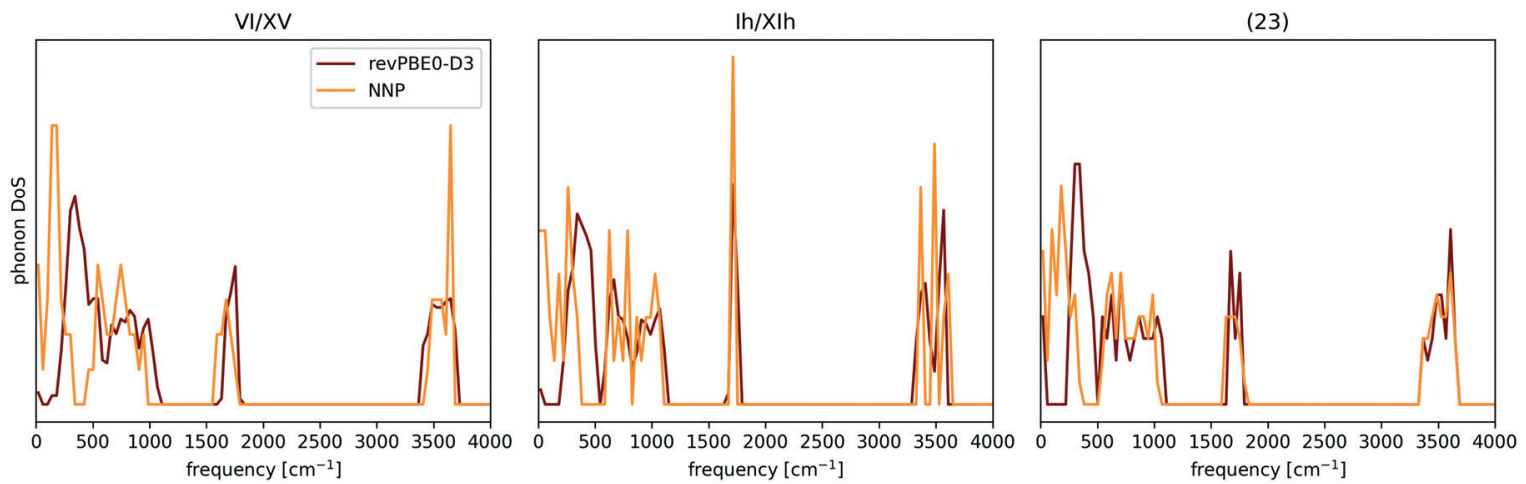

Fig. 6 Comparison between the NNP and revPBEO-D3 $\Gamma$-point phonon density of states (DOS) for proton-ordered realisations of ices VI and lh, and a low-density hypothetical phase. The discrepancies in the low frequencies are attributed to the importance of long-range interactions for long wavelength vibrational modes. The case of $\mathrm{lh}$ is of particular interest, since the NNP has proven to be an excellent surrogate potential for revPBEO-D3 level free energy calculations for ice Ih. The data was taken from ref. 137.
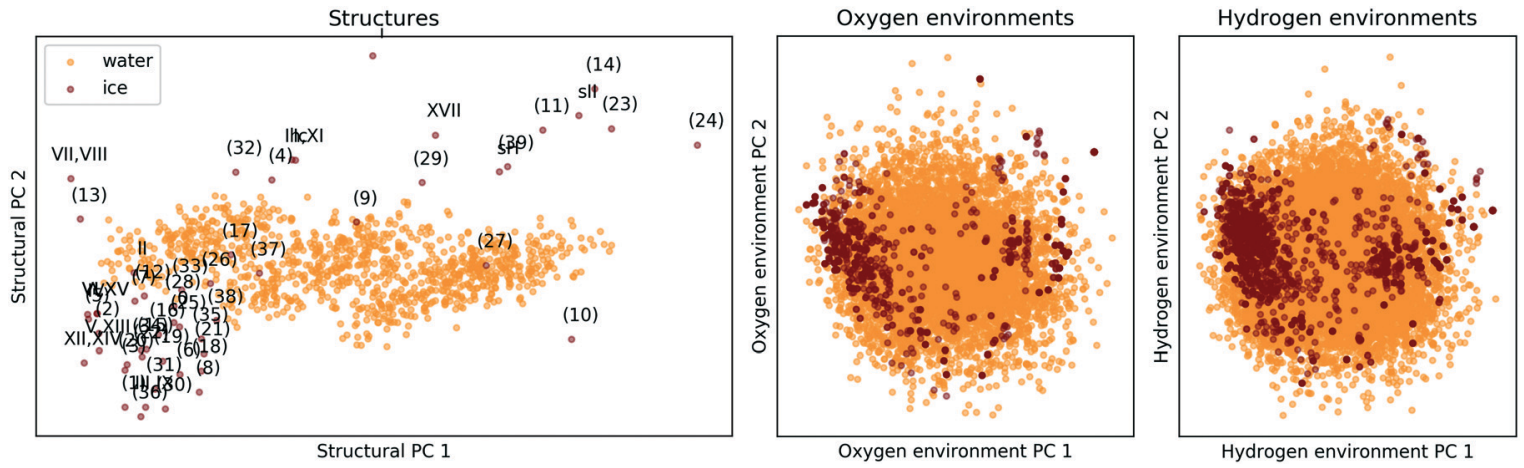

Fig. 7 PCA maps comparing the 53 ice polymorphs from section II C to 1000 snapshots of liquid water. The ice structure are labelled according to the scheme in Fig. 2. The left panel compares extended structures (i.e. the complete unit cells), while in the central and right panels oxygen or hydrogen-centered local environments are compared. The data was taken from ref. 137.

accurate NNP, orders of magnitude fewer reference calculations are required than would be required during direct statistical sampling of the nuclear degrees of freedom.

Starting from different proton arrangements consistent with the Bernal-Fowler ice rules is crucial, because (i) the proton order is effectively "frozen in" at the timescales available to simulation, ${ }^{134}$ even at temperature where ice I is proton-disordered, and (ii) differences in $\Delta G_{\mathrm{NN}}$ between such arrangements can be as large as several meV per molecule. ${ }^{61,121,135}$ Hence, different representative configurations (with zero net polarisation) were generated for each polymorph using the hydrogen-disordered ice generator ${ }^{136}$ to calculate $\Delta G_{\mathrm{cl}, \mathrm{NNN}}^{\mathrm{Ih} \rightarrow \mathrm{Ic}}$ and $\Delta G^{\mathrm{Ih} \rightarrow \mathrm{Ic}}$. Similarly, $G(p$, $T)-G_{\mathrm{NN}}(p, T)$ was determined as the average over multiple 64-molecule realisations of each polymorph.

Fig. 4 shows that $\Delta G_{\mathrm{cl}}^{\mathrm{Ih} \rightarrow \mathrm{Ic}}$ is negative, while the quantummechanical $\Delta G^{\mathrm{Ih} \rightarrow \mathrm{Ic}}$ is close to zero at $200-250 \mathrm{~K}$ and increases to $0.2 \pm 0.2 \mathrm{meV}$ per $\mathrm{H}_{2} \mathrm{O}$ at $300 \mathrm{~K}$, confirming the conclusion of ref. 61 that NQE are crucial in stabilizing the hexagonal form.

In more general terms, the above highlights the intricacies of determining relative stabilities of ice polymorphs and the value of surrogate ML potentials for predictive simulations of thermodynamic properties.
Of course, large scale application to the candidates from a (G) $\mathrm{CH}$ demands fairly universal applicability. The universality of the above hinges on that of the NNP, which in turn hinges on the decomposition of atomic configurations into local environments.

Indeed, the above NNP proves reasonably accurate for the 53 candidate structures from section II C. $\uparrow$ T To demonstrate this, the 53 candidates were geometry optimised at the revPBE0-D3 reference level of theory and harmonic vibrational calculations were performed as a simple measure of (nuclear) dynamics. Performing analogous calculations with the NNP shows that it accurately reproduces the reference configurational energies, densities, and vibrational density of states for the 53 ice phases ${ }^{137}$ (see Fig. 5 and 6).

To rationalise this universality the atomic structure of the 53 candidates is compared to that of 1000 structurally diverse snapshots of 64-molecule liquid water, which constitute part of the training data of the NNP. The latter are farthest-pointsampled $^{138}$ from simulations of bulk liquid water at high temperatures and densities between 0.7 and 1.2 gram per $\mathrm{ml}$ and subsequently relaxed to the corresponding local PES minima.

ๆๆ Three mixed phases and the very high pressure phase $\mathrm{X}$ were set aside. 
The comparison is again based on a SOAP description. A meaningful comparison of entire structures and local environments is rendered possible by a linear PCA projection, combined with constructing features of structures (global descriptors) as averages over those of the constituent atomic environments (local descriptors). The PCA map in the lefthand panel of Fig. 7 shows that the presence and absence of long-range order clearly distinguishes the 53 ice phases from the 1000 snapshots of liquid water, while the two righthand panels of Fig. 7 shows that the local atomic environments found in liquid water prototype all atomic environments pertinent to the 53 ice phases.||||

Like most common ML potentials, the above NNP exploits the notion of "nearsightedness", which implies that the energy and forces associated with any atom are largely determined by its neighbours, while long-range interactions can be approximated in a mean-field manner. ${ }^{139,140}$ The energetics and dynamics of extended systems are reconstructed from atom-centered energy contributions and forces, which only depend on local atomic environments. Consequently, the understanding of local properties encoded in the liquid water training data suffices for free energy calculations for general ice phases.

Notably, if such universal applicability cannot be achieved, assessing the uncertainty in the ML predictions allows implementing either an active learning strategy ${ }^{124}$ or a baselining procedure, ${ }^{141}$ to ensure universally meaningful energy and force predictions.

With an understanding of thermodynamic stability, one may now consider establishing possible synthesis pathways, using approaches such as forward flux sampling, ${ }^{142}$ or via the identification of suitable collective variables. ${ }^{143,144}$

\section{Conclusions and open problems}

Ref. 55 probably constitutes the most extensive survey of (meta-)stable, crystalline phases of ice to date. Yet, the recent discoveries of stable low-density forms ${ }^{48,67,68,145}$ suggest that our understanding of the $p-T$ phase-diagram is probably still incomplete. The general phase diagram, including thermodynamic and geometric constraints such as electric fields or substrates, is even less well established. Furthermore, the observed properties of ice are not fully determined by its ideal, pure, crystalline forms, but reflect the extensive presence of defects, ranging from point-defects like hydrogen bonding/Bjerrum defects ${ }^{146}$ and atomic substitutions, $^{64}$ to extended defects like stackingdisorder ${ }^{147-149}$ and grain boundaries ${ }^{150}$ and surfaces. ${ }^{151}$ Their nature and extent pertains to the stability of and transitions between phases and thus our understanding of the space of crystalline phases of water, but has been studied much more sparsely (at least in computational science).

|||| The ordered nature of the ice structures is reflected in comparatively few distinct atomic environments.
The above outlines how data-driven approaches facilitate the extensive survey of synthesisable crystalline phases of water. In the process it highlights three important challenges, in particular, and suggests transferable strategies for their resolution. The first concerns the (potentially extensive) space of candidate structures generated at the outset of a CMD workflow. Given that the number of candidates is typically far too large to permit developing an understanding by visual inspection of individual candidates, the above proposes using suitable measures of structural similarity coupled to dimensionality reduction algorithms to extract the key distinguishing structural features, thereby rendering large numbers of candidates comparable. The second challenge is that of screening the thus rationalised structure space for candidates with an appreciable chance of being experimentally realisable. A generalised convex hull construction is thus used to gauge stability at general thermodynamic conditions. The final challenge lies in putting the resultant understanding of thermodynamic stability for a potentially still appreciable number of candidates on a more solid footing. The above highlights that the use of ML surrogate potentials renders it feasible to perform accurate and extensive free energy calculations for significant numbers of candidates to more rigorously assess phase behaviour. It moreover highlights that suitable constructed ML models provide a sufficiently transferable basis for doing so.

While the above tricks of the trade can in principle be applied to "design" new materials by uncovering phases with novel and/or valuable properties, it is worth emphasising that it does not yet constitute a universal CMD framework. First and foremost, the identification of stabilisation mechanisms has barely been touched upon and the characterisation of properties and possible synthesis pathways has been set aside entirely. Second, there are obvious caveats to its universality. For instance, stabilisable structures which are unstable at the conditions of the initial structure search will escape identification, as will structures that are dynamically/ entropically stabilised. The latter constitute an important and promising class of materials. ${ }^{152}$ Moreover, the above approach relies on the availability of (i) an accurate description of electronic structure of the system of interest and (ii) the accuracy of surrogate potentials, which treat longrange interactions in a mean-field manner in return for the ability to generalise across different polymorphs/ conformations.

Last but not least, the availability of a usable, integrated package is the key to CMD fulfilling its potential. This has not been addressed, although atomic structure-, workflow-, and data-management tools/platforms such as ASE, ${ }^{153}$ AiiDa $^{154,155}$ and the Materials Cloud ${ }^{156}$ provide an excellent basis.

\section{Conflicts of interest}

There are no conflicts to declare. 


\section{Acknowledgements}

EAE acknowledges financial support from Trinity College, Cambridge. The original work was performed with support of the Engineering and Physical Sciences Research Council of the UK [EP/J017639/1], the European Research Council under the European Union's Horizon 2020 research and innovation programme [677013-HBMAP], and the NCCR MARVEL, funded by the Swiss National Science Foundation (SNSF).

\section{Notes and references}

1 N. Metropolis, A. W. Rosenbluth, M. N. Rosenbluth and A. H. Teller, J. Chem. Phys., 1953, 21, 1087.

2 C. J. Pickard, M. Martinez-Canales and R. J. Needs, Phys. Rev. B, 2012, 85, 214114.

3 S. Azadi, B. Monserrat, W. M. Foulkes and R. J. Needs, Phys. Rev. Lett., 2014, 112, 165501.

4 N. D. Drummond, B. Monserrat, J. H. Lloyd-Williams, P. López Ríos, C. J. Pickard and R. J. Needs, Nat. Commun., 2015, 6, 7794.

5 I. Errea, M. Calandra, C. J. Pickard, J. R. Nelson, R. J. Needs, Y. Li, H. Liu, Y. Zhang, Y. Ma and F. Mauri, Phys. Rev. Lett., 2015, 114, 157004.

6 A. P. Drozdov, M. I. Eremets, I. A. Troyan, V. Ksenofontov and S. I. Shylin, Nature, 2015, 525, 73.

7 M. Mayo, K. J. Griffith, C. J. Pickard and A. J. Morris, Chem. Mater., 2016, 28, 2011.

8 B. Monserrat, R. J. Needs, E. Gregoryanz and C. J. Pickard, Phys. Rev. B, 2016, 94, 134101.

9 C. J. Pickard and R. J. Needs, Phys. Rev. Lett., 2006, 97, 045504.

10 C. W. Glass, A. R. Oganov and N. Hansen, Comput. Phys. Commun., 2006, 175, 713.

11 M. Amsler and S. Goedecker, J. Chem. Phys., 2010, 133, 224104.

12 T.-Q. Yu and M. E. Tuckerman, Phys. Rev. Lett., 2011, 107, 015701.

13 Q. Zhu, A. R. Oganov, C. W. Glass and H. T. Stokes, Acta Crystallogr., Sect. B: Struct. Sci., 2012, 68, 215.

14 S. P. Ong, W. D. Richards, A. Jain, G. Hautier, M. Kocher, S. Cholia, D. Gunter, V. L. Chevrier, K. A. Persson and G. Ceder, Comput. Mater. Sci., 2013, 68, 314.

15 A. M. Reilly, et al., Acta Crystallogr., Sect. B: Struct. Sci., Cryst. Eng. Mater., 2016, 72, 439.

16 T. H. Nguyen and S. K. O’Leary, J. Appl. Phys., 2000, 88, 3479.

17 E. M. Thomas, B. C. Popere, H. Fang, M. L. Chabinyc and R. A. Segalman, Chem. Mater., 2018, 30, 2965.

18 F. S. A. Fediai, A. Emering and W. Wenzel, Phys. Chem. Chem. Phys., 2020, 22, 10256.

19 E. P. George, D. Raabe and R. O. Ritchie, Nat. Rev. Mater., 2019, 4, 515.

20 B. Pamuk, J. M. Soler, R. Ramírez, C. P. Herrero, P. W. Stephens, P. B. Allen and M. V. Fernandez-Serra, Phys. Rev. Lett., 2012, 108, 193003.

21 P. Bridgman, J. Chem. Phys., 1935, 3, 597.
22 K. Röttger, A. Endriss, J. Ihringer, S. Doyle and W. F. Kuhs, Acta Crystallogr., Sect. B: Struct. Sci., 1994, 50, 644.

23 A. Tkatchenko, R. A. Di Stasio, R. Car and M. Scheffler, Phys. Rev. Lett., 2012, 108, 236402.

24 R. A. DiStasio Jr., O. A. von Lilienfeld and A. Tkatchenko, Proc. Natl. Acad. Sci. U. S. A., 2012, 109, 14791.

25 N. Marom, R. A. DiStasio Jr., V. Atalla, S. Levchenko, A. M. Reilly, J. R. Chelikowsky, L. Leiserowitz and A. Tkatchenko, Am. Ethnol., 2013, 52, 6629.

26 J. Hermann, R. A. DiStasio Jr. and A. Tkatchenko, Chem. Rev., 2017, 117, 4714.

27 A. Geim and I. Grigorieva, Nature, 2013, 499, 419.

28 L. del Rosso, M. Celli, F. Grazzi, M. Catti, T. C. Hansen, A. D. Fortes and L. Ulivi, Nat. Mater., 2020, 19, 663.

29 K. Komatsu, S. Machida, F. Noritake, T. Hattori, A. SanoFurukawa, R. Yamane, K. Yamashita and H. Kagi, Nat. Commun., 2020, 11, 464.

30 C. G. Salzmann and B. J. Murray, Nat. Mater., 2020, 19, 581.

31 R. Blinc, J. Phys. Chem. Solids, 1960, 13, 204.

32 S. Crawford, Angew. Chem., Int. Ed., 2009, 48, 755.

33 T. D. Nguyen, et al., Nat. Mater., 2010, 9, 345.

34 P. V. Hobbs, Ice physics, Oxford University Press, Oxford, 2010.

35 V. F. Petrenko and R. W. Whitworth, Physics of Ice, Oxford University Press, Oxford, 1999.

36 J. D. Bernal and R. H. Fowler, J. Chem. Phys., 1933, 1, 515.

37 R. J. N. Baldock, N. Bernstein, K. M. Salerno, L. B. Pártay and G. Csányi, Phys. Rev. E, 2017, 96, 043311.

38 P. M. Piaggi and M. Parrinello, J. Chem. Phys., 2019, 150, 244119.

39 D. Quigley and P. M. Rodger, Mol. Simul., 2009, 35, 613.

40 F. Giberti, et al., IUCrJ, 2015, 2, 256.

41 S. Curtarolo, G. L. W. Hart, M. B. Nardelli, N. Mingo, S. Sanvito and O. Levy, Nat. Mater., 2013, 12, 191.

42 A. R. Oganov, C. J. Pickard and Q. Zhu, et al., Nat. Rev. Mater., 2019, 4, 331.

43 C. J. Pickard, M. Martinez-Canales and R. J. Needs, Phys. Rev. Lett., 2013, 110, 245701.

44 J. Hama and K. Suito, On metallization of ice under ultra-high pressures, In Physics and chemistry of ice, ed. N. Maeno and T. Hondoh, Hokkaido University Press, Sapporo, 1992.

45 C. J. Pickard, M. Martinez-Canales and R. J. Needs, Phys. Rev. Lett., 2013, 110, 245701.

46 J. Russo, F. Romano and H. Tanaka, Nat. Mater., 2014, 13, 733.

47 C. J. Fennell and J. D. Gezelter, J. Chem. Theory Comput., 2005, 1, 662 .

48 Y. Huang, C. Zhu, L. Wang, J. Zhao and X. C. Zeng, Chem. Phys. Lett., 2017, 671, 186.

49 T. Matsui, M. Hirata, T. Yagasaki, M. Matsumoto and H. Tanaka, J. Chem. Phys., 2017, 147, 091101.

50 I. A. Baburin, D. M. Proserpio, V. A. Saleev and A. V. Shipilova, Phys. Chem. Chem. Phys., 2015, 17, 1332.

51 G. A. Tribello, B. Slater, M. A. Zwijnenburg and R. G. Bell, Phys. Chem. Chem. Phys., 2010, 12, 8597. 
52 J. Emmer and M. Wiebcke, J. Chem. Soc., Chem. Commun., 1994, 2079.

53 M. Wiebcke, J. Chem. Soc., Chem. Commun., 1991, 1507.

54 M. Wiebcke, J. Emmer and J. Felsche, J. Chem. Soc., Chem. Commun., 1993, 1604.

55 E. A. Engel, A. Anelli, M. Ceriotti, C. J. Pickard and R. J. Needs, Nat. Commun., 2018, 9, 2173.

56 C. Baerlocher, W. M. Meier and D. H. Olson, Atlas of Zeolite Framework Types, Elsevier, Amsterdam, 2007.

57 M. M. J. Treacy, I. Rivin, E. Balkovsky, K. H. Randall and M. D. Foster, Microporous Mesoporous Mater., 2004, 74, 121.

58 D. J. Earl and M. W. Deem, Ind. Eng. Chem. Res., 2006, 45, 5449.

59 C. Baerlocher and L. McCusker, Database of zeolite structures, http://www.iza-structure.org/databases/, accessed: 28/06/2017.

60 A. C. T. van Duin, S. Dasgupta, F. Lorant and W. A. G. III, J. Phys. Chem. A, 2001, 105, 9396.

61 E. A. Engel, B. Monserrat and R. J. Needs, Phys. Rev. X, 2015, 5, 021033.

62 J. P. Perdew, K. Burke and M. Ernzerhof, Phys. Rev. Lett., 1996, 77, 3865

63 A. Pulido, L. Chen, T. Kaczorowski, D. Holden, M. A. Little, S. Y. Chong, B. J. Slater, D. P. McMahon, B. Bonillo, C. J. Stackhouse, A. Stephenson, C. M. Kane, R. Clowes, T. Hasell, A. I. Cooper and G. M. Day, Nature, 2017, 657, 543.

64 H. Fukazawa, S. Ikeda and S. Mae, Chem. Phys. Lett., 1998, 282, 215.

65 G. A. Tribello and B. Slater, J. Chem. Phys., 2009, 131, 024703.

66 A. Falenty, T. C. Hansen and W. F. Kuhs, Nature, 2014, 516, 231.

67 L. del Rosso, F. Grazzi, M. Celli, D. Colognesi, V. GarciaSakai and L. Ulivi, J. Phys. Chem. C, 2016, 120, 26955.

68 L. del Rosso, M. Celli and L. Ulivi, Nat. Commun., 2016, 7, 13394.

69 A. Grisafi, D. M. Wilkins, G. Csányi and M. Ceriotti, Phys. Rev. Lett., 2018, 120, 036002.

70 M. Rupp, A. Tkatchenko, K.-R. Müller and O. A. von Lilienfeld, Phys. Rev. Lett., 2012, 108, 058301.

71 K. Hansen, F. Biegler, R. Ramakrishnan, W. Pronobis, O. A. Von Lilienfeld, K.-R. Müller and A. Tkatchenko, J. Phys. Chem. Lett., 2015, 6, 2326.

72 B. Huang and A. von Lilienfeld, 2019, arXiv:1707.04146.

73 J. Behler and M. Parrinello, Phys. Rev. Lett., 2007, 98, 146401.

74 A. P. Bartók, M. J. Gillan, F. R. Manby and G. Csányi, Phys. Rev. B, 2013, 88, 054104.

75 S. De, A. P. Bartók, G. Csányi and M. Ceriotti, Phys. Chem. Chem. Phys., 2016, 18, 13754.

76 M. J. Willatt, F. Musil and M. Ceriotti, J. Chem. Phys., 2019, 150, 154110.

77 S. N. Pozdnyakov, M. J. Willatt, A. P. Bartók, C. Ortner, G. Csányi and M. Ceriotti, 2020, arXiv:2001.11696.

78 M. E. Tipping and C. M. Bishop, J. R. Stat. Soc. Series B Stat. Methodol., 1999, 61, 611.
79 B. Schölkopf, A. J. Smola and K.-R. Müller, in Advances in kernel methods, MIT Press, Cambridge, MA, USA, 1999, p. 327.

80 L. McInnes and J. Healy, 2018, arXiv:1802.03426.

81 L. J. P. van der Maaten and G. E. Hinton, J. Mach. Learn. Res., 2008, 9, 2579.

82 M. Ceriotti, G. A. Tribello and M. Parrinello, Proc. Natl. Acad. Sci. U. S. A., 2011, 108, 13023.

83 H. Niu, A. R. Oganov, X.-Q. Chen and D. Li, Sci. Rep., 2015, 5, 18347.

84 R. Malik, F. Zhou and G. Ceder, Nat. Mater., 2011, 10, 587.

85 G. Algara-Siller, O. Lehtinen, F. C. Wang, R. R. Nair, U. Kaiser, H. A. Wu, A. K. Geim and I. V. Grigorieva, Nature, 2015, 519, 443.

86 D. Takaiwa, I. Hatano, K. Koga and H. Tanaka, Proc. Natl. Acad. Sci. U. S. A., 2008, 105, 39.

87 A. Anelli, E. A. Engel, C. J. Pickard and M. Ceriotti, Phys. Rev. Mater., 2018, 2, 103804.

88 K. Fukunaga and D. R. Olsen, IEEE Trans. Comput., 1971, 20, 176.

89 W. Sun, et al., Sci. Adv., 2016, 2, e1600225.

90 V. Stevanovic, Phys. Rev. Lett., 2016, 116, 075503.

91 M. Born and R. Oppenheimer, Ann. Phys., 1927, 389, 457.

92 K. Lejaeghere, G. Bihlmayer, T. Björkman, P. Blaha, S. Blügel, V. Blum, D. Caliste, I. E. Castelli, S. J. Clark, A. D. Corso, S. de Gironcoli, T. Deutsch, J. K. Dewhurst, I. D. Marco, C. Draxl, M. Dułak, O. Eriksson, J. A. Flores-Livas, K. F. Garrity, L. Genovese, P. Giannozzi, M. Giantomassi, S. Goedecker, X. Gonze, O. Grånäs, E. K. U. Gross, A. Gulans, F. Gygi, D. R. Hamann, P. J. Hasnip, N. A. W. Holzwarth, D. Iuşan, D. B. Jochym, F. Jollet, D. Jones, G. Kresse, K. Koepernik, E. Küçükbenli, Y. O. Kvashnin, I. L. M. Locht, S. Lubeck, M. Marsman, N. Marzari, U. Nitzsche, L. Nordström, T. Ozaki, L. Paulatto, C. J. Pickard, W. Poelmans, M. I. J. Probert, K. Refson, M. Richter, G. Rignanese, S. Saha, M. Scheffler, M. Schlipf, K. Schwarz, S. Sharma, F. Tavazza, P. Thunström, A. Tkatchenko, M. Torrent, D. Vanderbilt, M. J. van Setten, V. V. Speybroeck, J. M. Wills, J. R. Yates, G. Zhang and S. Cottenier, Science, 2016, 351, 6280.

93 B. M. Austin, D. Y. Zubarev and W. A. Lester, Chem. Rev., 2012, 112, 263.

94 G. Onida, L. Reining and A. Rubio, Rev. Mod. Phys., 2002, 74, 601.

95 G. Kotliar, S. Y. Savrasov, K. Haule, V. S. Oudovenko, O. Parcollet and C. A. Marianetti, Rev. Mod. Phys., 2006, 78, 865.

96 G. Rohringer, H. Hafermann, A. Toschi, A. A. Katanin, A. E. Antipov, M. I. Katsnelson, A. I. Lichtenstein, A. N. Rubtsov and K. Held, Rev. Mod. Phys., 2018, 90, 025003.

97 F. Neese, M. Atanasov, G. Bistoni, D. Maganas and S. Ye, J. Am. Chem. Soc., 2019, 141, 2814.

98 C. Riplinger, P. Pinski, U. Becker, E. F. Valeev and F. Neese, J. Chem. Phys., 2016, 144, 024109.

99 T. Gruber, K. Liao, T. Tsatsoulis, F. Hummel and A. Grüneis, Phys. Rev. X, 2018, 8, 021043. 
100 E. Caldeweyher and J. G. Brandenburg, J. Phys.: Condens. Matter, 2018, 30, 213001.

101 A. Zen, J. G. Brandenburg, J. Klimeš, A. Tkatchenko, D. Alfè and A. Michaelides, Proc. Natl. Acad. Sci. U. S. A., 2018, 115, 1724.

102 J. Lekner, Phys. B, 1998, 252, 149.

103 S. Habershon, T. E. Markland and D. E. Manolopoulos, J. Chem. Phys., 2009, 131, 024501.

104 R. Ramírez and C. P. Herrero, J. Chem. Phys., 2010, 133, 144511.

105 R. Ramírez, N. Neuerburg and C. P. Herrero, J. Chem. Phys., 2012, 137, 134503.

106 B. Cheng, J. Behler and M. Ceriotti, J. Phys. Chem. Lett., 2016, 7, 2210.

107 R. Ramírez, N. Neuerburg, M.-V. Fernández-Serra and C. P. Herrero, J. Chem. Phys., 2012, 137, 044502.

108 V. Kapil, E. A. Engel, M. Rossi and M. Ceriotti, J. Chem. Theory Comput., 2019, 15, 5845.

109 J. G. Kirkwood, J. Chem. Phys., 1935, 3, 300.

110 D. Chandler and P. G. Wolynes, J. Chem. Phys., 1981, 74, 4078.

111 M. Parrinello and A. Rahman, J. Chem. Phys., 1984, 80, 860.

112 L. M. Ghiringhelli, J. H. Los, E. J. Meijer, A. Fasolino and D. Frenkel, Phys. Rev. Lett., 2005, 94, 145701.

113 M. Tuckerman, Statistical Mechanics: Theory and Molecular Simulation, Oxford University Press, Oxford, UK, 2010.

114 B. Cheng and M. Ceriotti, J. Chem. Phys., 2014, 141, 244112.

115 B. Cheng, E. A. Engel, J. Behler, C. Dellago and M. Ceriotti, Proc. Natl. Acad. Sci. U. S. A., 2019, 116, 1110.

116 Y. Zhang and W. Yang, Phys. Rev. Lett., 1998, 80, 890.

117 C. Adamo and V. Barone, J. Chem. Phys., 1999, 110, 6158.

118 L. Goerigk and S. Grimme, Phys. Chem. Chem. Phys., 2011, 13, 6670.

119 S. Grimme, J. Antony, S. Ehrlich and S. Krieg, J. Chem. Phys., 2010, 132, 154104.

120 O. Marsalek and T. E. Markland, J. Phys. Chem. Lett., 2017, 8, 1545.

121 Z. Raza, D. Alfè, C. G. Salzmann, J. Klimeš, A. Michaelides and B. Slater, Phys. Chem. Chem. Phys., 2011, 13, 19788.

122 M. Macher, J. Klimeš, C. Franchini and G. Kresse, J. Chem. Phys., 2014, 140, 084502.

123 F. Musil, M. J. Willatt, M. A. Langovoy and M. Ceriotti, J. Chem. Theory Comput., 2019, 15, 906.

124 E. V. Podryabinkin and A. V. Shapeev, Comput. Mater. Sci., 2017, 140, 171.

125 N. Bernstein, G. Csányi and V. L. Deringer, npj Comput. Mater., 2019, 5, 99.

126 R. Tibshirani, J. R. Stat. Soc. Series B Stat. Methodol, 1996, 58, 267.

127 B. D. Conduit, N. G. Jones, H. J. Stone and G. J. Conduit, Mater. Des., 2017, 131, 358.

128 P. C. Verpoort, P. MacDonald and G. J. Conduit, Comput. Mater. Sci., 2018, 147, 176.

129 J. Behler, Angew. Chem., Int. Ed., 2017, 56, 12828.

130 T. Morawietz, A. Singraber, C. Dellago and J. Behler, Proc. Natl. Acad. Sci. U. S. A., 2016, 113, 8368.

131 B. Cheng and M. Ceriotti, Phys. Rev. B, 2018, 97, 054102.
132 M. Ceriotti and T. E. Markland, J. Chem. Phys., 2013, 138, 014112.

133 B. Cheng, A. T. Paxton and M. Ceriotti, Phys. Rev. Lett., 2018, 120, 225901.

134 C. Drechsel-Grau and D. Marx, Phys. Rev. Lett., 2014, 112, 148302.

135 S. J. Singer and C. Knight, in Advances in Chemical Physics, ed. S. A. Rice and A. R. Dinner, Wiley \& Sons, Inc, Hoboken, NJ, USA, 2011, vol. 147.

136 M. Matsumoto, T. Yagasaki and H. Tanaka, J. Comput. Chem., 2018, 39, 61.

137 B. Monserrat, J. G. Brandenburg, E. A. Engel and B. Cheng, Nat. Commun., 2020, 11, 5757.

138 Y. Eldar, M. Lindenbaum, M. Porat and Y. Y. Zeevi, IEEE Trans. Image Process., 1997, 6, 1305.

139 W. Kohn, Phys. Rev. Lett., 1996, 76, 3168.

140 J. Behler, J. Chem. Phys., 2016, 145, 170901.

141 G. Imbalzano, Y. Zhuang, V. Kapil, K. Rossi, E. A. Engel, F. Grasselli and M. Ceriotti, 2020, arXiv:2011.08828 [physics. chem-ph].

142 R. J. Allen, et al., J. Chem. Phys., 2006, 124, 024102.

143 V. Rizzi, D. Mendels, E. Sicilia and M. Parrinello, J. Chem. Theory Comput., 2019, 15, 4507.

144 L. Bonati, V. Rizzi and M. Parrinello, J. Phys. Chem. Lett., 2020, 11, 2998.

145 Y. Huang, C. Zhu, L. Wang, X. Cao, Y. Su, X. Jiang, S. Meng, J. Zhao and X. C. Zeng, Sci. Adv., 2016, 2, e1501010.

146 P. J. Wooldridge, H. H. Richardson and J. P. Devlin, J. Chem. Phys., 1987, 87, 4126.

147 E. B. Moore and V. Molinero, Phys. Chem. Chem. Phys., 2011, 13, 20008.

148 W. F. Kuhs, C. Sippel, A. Falenty and T. C. Hansen, Proc. Natl. Acad. Sci. U. S. A., 2012, 109, 21259.

149 T. L. Malkin, B. J. Murray, C. G. Salzmann, V. Molinero, S. J. Pickering and T. F. Whale, Phys. Chem. Chem. Phys., 2015, 17, 60.

150 P. A. F. P. Moreira, R. G. de Aguiar Veiga, I. de Almeida Ribeiro, R. Freitas, J. Helfferich and M. de Koning, Phys. Chem. Chem. Phys., 2018, 20, 13944.

151 M. Watkins, D. Pan, E. G. Wang, A. Michaelides, J. VandeVondele and B. Slater, Nat. Mater., 2011, 10, 794.

152 C. Toher, C. Oses, D. Hicks and S. Curtarolo, npj Comput. Mater., 2019, 5, 69.

153 A. H. Larsen, J. J. Mortensen, J. Blomqvist, I. E. Castelli, R. Christensen, M. Dułak, J. Friis, M. N. Groves, B. Hammer, C. Hargus, E. D. Hermes, P. C. Jennings, P. B. Jensen, J. Kermode, J. R. Kitchin, E. L. Kolsbjerg, J. Kubal, K. Kaasbjerg, S. Lysgaard, J. B. Maronsson, T. Maxson, T. Olsen, L. Pastewka, A. Peterson, C. Rostgaard, J. Schiøtz, O. Schütt, M. Strange, K. S. Thygesen, T. Vegge, L. Vilhelmsen, M. Walter, Z. Zeng and K. W. Jacobsen, J. Phys.: Condens. Matter, 2017, 29, 273002.

154 G. Pizzi, A. Cepellotti, R. Sabatini, N. Marzari and B. Kozinsky, Comput. Mater. Sci., 2016, 111, 218.

155 S. P. Huber, S. Zoupanos, M. U. L. Talirz, L. Kahle, R. Häuselmann, D. Gresch, T. Müller, A. V. Yakutovich, C. W. Andersen, F. F. Ramirez, C. S. Adorf, F. Gargiulo, S. Kumbhar, E. Passaro, C. Johnston, A. Merkys, A. Cepellotti, 
N. Mounet, N. Marzari, B. Kozinsky and G. Pizzi, Sci. Data, 2020, 7, 300.

156 L. Talirz, S. Kumbhar, E. Passaro, A. V. Yakutovich, V. Granata, F. Gargiulo, M. Borelli, M. Uhrin, S. P. Huber, S.
Zoupanos, C. S. Adorf, C. W. Andersen, O. Schütt, C. A. Pignedoli, D. Passerone, J. VandeVondele, T. C. Schulthess, B. Smit, G. Pizzi and N. Marzari, Sci. Data, 2020, 7, 299. 